\title{
Non-overlapping domain decomposition scheme for the symmetric radial basis function meshless approach with double collocation at the sub-domain interfaces
}

\author{
H. Power, A. Hernandez \& A. La Rocca \\ The University of Nottingham, \\ School of Mechanical, Material and Manufacturing Engineering, UK
}

\begin{abstract}
In the particular case of solving large-scale boundary value problems, the computational cost derived as a result of the application of any numerical scheme represents a determinant factor in the determination of its computational efficiency. The present work studies the influence of the non-overlapping domain decomposition on the symmetric radial basis collocation method, as a way to improve its efficiency under high demanding numerical conditions. Due to the Hermitian character of the symmetric scheme at each of the collocations points of the sub-domain interfaces it is possible to impose simultaneously all the corresponding matching conditions. A multi-zone problem is considered as a test example, comparison between the numerical result and the analytical solution for two set of different physical parameters are presented.
\end{abstract}

Keywords: symmetric RBF meshless approach, domain decomposition and double collocation.

\section{Introduction}

The use of a mesh is a basic characteristic of traditional numerical approaches for the solution of partial differential equations, as is the case of the finite difference, element, volume and the boundary element methods. In the first cases, assumptions are made for the local approximation, which require internal mesh to support them. On the other hand, in the case on boundary methods, a 
boundary mesh is required to obtain a numerical approximation of the resulting boundary integrals.

During recent years, considerable effort has been given to the development of the so-called free-mesh methods (meshless approach). The aim of this type of approach is to eliminate at least the structure of the mesh and approximate the solution entirely using nodes values inside and/or in the boundary quasi random distributed in the domain.

Recently, some significant developments in meshless methods for solving boundary value problems of partial differential equations have been reported in the literature. Kansa [1, 2] introduced the concept of solving PDEs using radial basis functions (RBFs) (Unsymmetric scheme). This type of approach, which approximates the whole solution of the PDE directly using RBFs, is very attractive due to the fact that this is truly a mesh free technique.

The Kansa's method has been applied successfully in several cases (see for example [3-5]). However, no existence of solution and convergence analysis is available in the literature and for some cases, it has been reported that the resulting matrix was extremely ill-conditioned and even singular for some distribution of the nodal points (see [6]).

Several techniques have been proposed to improve the conditioning of the coefficient matrix and the solution accuracy, as are: the use of high order interpolation functions, replacement of global solvers by block partitioning, LU decomposition schemes, matrix preconditioners, overlapping and nonoverlapping domain decomposition etc (see [7]). Fedoseyev et al. [8] proposed the use of a set of additional nodes at the boundary and beyond the boundary (at the exterior) where the governing equation is required to be satisfied. It was found that the suggested approach yields to more accurate results than only imposing the governing equation at internal nodes.

Fasshauer [9] suggested an alternative approach to the Unsymmetric scheme based on the Hermite interpolation property of the radial basis functions, which states that the RBFs not only are able to interpolate a given function but also its derivatives. The convergence proof for RBF Hermite-Brikhoff interpolation was given by $\mathrm{Wu}$ [10] who also proved the convergence of this approach when solving PDEs (see Wu [11] and Schaback and Franke [12]). Another advantage of the Hermite based approach is that the matrix resulting from the scheme is symmetric, as opposed to the completely unstructured matrix of the same size resulting from Unsymmetric schemes.

The main objective of this work is to study and test some of the above mentioned techniques previously used to improve the efficiency of the Unsymmetric approach in order to increase the computational efficiency of the radial basis function symmetric approach. In particular, we will study the nonoverlapping domain decomposition with a double collocation at the sub-domain interfaces. The domain decomposition approach is itself a very powerful and popular scheme in numerical analysis, which have recently increased its popularity due to its use in parallel computing algorithms. 


\section{Symmetric radial basis function meshless approach}

Let us consider a boundary value problem defined by:

$$
\begin{aligned}
& L[C](x)=f(x) \\
& B[C](x)=g(x)
\end{aligned}
$$

where the operators $\mathrm{L}$ and $\mathrm{B}$ are linear partial differential operators on the domain $\Omega$ and at the contour $\Gamma$ respectively.

A symmetric RBF collocation method (Fasshauer [9]), represents the solution of the above boundary value problem by the interpolation function:

$$
C(x)=\sum_{k=1}^{n} \lambda_{k} B_{\xi} \Psi\left(\left\|x-\xi_{k}\right\|\right)+\sum_{k=n+1}^{N} \lambda_{k} L_{\xi} \Psi\left(\left\|x-\xi_{k}\right\|\right)+P_{m-1}(x)
$$

with $n$ as the number of nodes on the boundary of $\Omega$ and $N-n$ the number of internal nodes. Here, $\Psi\left(\left\|x-x_{j}\right\|\right)$ is a conditionally positive definite RBF of order $m$ and $\mathrm{P}$ a polynomial term of order $m-1$.

In the above expression $L_{\xi}$ and $B_{\xi}$ are the differential operators used in (1-a,b), but acting on $\Psi$ viewed as a function of the second argument $\xi$ (see Fasshauer [9]). This expansion for $C$ leads to a collocation matrix A, which is of the form

$$
A=\left(\begin{array}{ccc}
B_{x} B_{\xi}[\Psi] & B_{x} L_{\xi}[\Psi] & B_{x} P_{m-1} \\
L_{x} B_{\xi}[\Psi] & L_{x} L_{\xi}[\Psi] & L_{x} P_{m-1} \\
B_{x} P_{m-1}^{T} & L_{x} P_{m-1}^{T} & 0
\end{array}\right)
$$

where the following ortogonality conditions is required to complete the system:

$$
\sum_{k=1}^{n} \lambda_{k} B_{x} P_{m-1}^{T}+\sum_{k=n+1}^{N} \lambda_{k} P_{m-1}^{T}=0
$$

The matrix (3) is of the same type as the scattered Hermite interpolation matrices and thus non-singular as long as $\Psi$ is chosen appropriately (see $\mathrm{Wu}[6]$ ). A major point in favour of the Hermite based approach is that the matrix resulting from the scheme is symmetric, as opposed to the completely unstructured matrix of the same size resulting from Unsymmetric schemes.

\section{Convection-diffusion problem}

The steady state differential equation considered in this work is of the form:

$$
D \frac{d^{2} C}{d x_{j}^{2}}-u_{j} \frac{d C}{d x_{j}}+k C=0
$$

The partial differential operators on the matrix representation (3) of the symmetric collocation numerical solution of equation (5), when satisfying 
boundary conditions of the first and second kind (Dirichlet and Neumann), are defined by the following expressions:

$$
\begin{aligned}
& L_{x}=D \frac{d^{2}}{d x_{j}^{2}}-u_{j} \frac{d}{d x_{j}}+k, L_{\xi}=D \frac{d^{2}}{d \xi_{j}^{2}}-u_{j} \frac{d}{d \xi_{j}}+k, \\
& B_{x}^{D}=1, B_{x}^{N}=\frac{d}{d x_{j}} n_{j}(x), B_{\xi}^{D}=1, B_{\xi}^{D}=\frac{d}{d \xi_{j}} n_{j}(\xi)
\end{aligned}
$$

In the above relations the super index $\mathrm{D}$ and $\mathrm{N}$ in the operator $\mathrm{B}$ represent the type of boundary conditions implemented, i.e. Dirichlet and Neumann.

In this work we will use the generalized TPS. Furthermore to avoid singularity at $r=0$ on the resulting differential operators of the matrix A, we use in the representation formula (2) the generalized TPS

$$
\psi=r^{6} \log r
$$

together with the corresponding cubic polynomial.

$$
\begin{gathered}
P_{3}(x)=\lambda_{N+1} x_{1}^{3}+\lambda_{N+2} x_{2}^{3}+\lambda_{N+3} x_{1}^{2} x_{2}+\lambda_{N+4} x_{1} x_{2}^{2}+\lambda_{N+5} x_{1}^{2}+ \\
\lambda_{N+6} x_{2}^{2}+\lambda_{N+7} x_{1} x_{2}+\lambda_{N+8} x_{1}+\lambda_{N+9} x_{2}+\lambda_{N+10}
\end{gathered}
$$

\section{Domain decomposition approach}

Domain decomposition methods are frequently used in two contexts. First: the division of problems into smaller problems usually through artificial subdivisions of the domain, as a way to improve the performance of a numerical technique. Second, many problems involve more than one mathematical model each posed on a different domain, so that domain decomposition occurs naturally.

When dealing with the numerical simulation of large problems, it is usual to use the method of domain decomposition, in which the original domain is divided into sub-regions, and on each of them the original governing equations are imposed.

The main objective of the domain decomposition method is to decompose one large global problem into smaller sub-domain problems. In the implementation of the domain decomposition approach, two different alternatives are possible to use: overlapping and non-overlapping schemes.

In the non-overlapping technique, the domain is divided into non-overlapping sub-domains having common interface surfaces. In each sub-domain the original numerical scheme is implemented. Owing to the lack of the boundary condition on the interface between sub-domains, additional surface unknowns need to be determined, i.e. in the present case the value of the concentration and the surface flux. For each interface boundary point, the number of unknowns is more than the number of the equations and therefore the resulting system is underdetermined. However, once the matching conditions are imposed and the sub-domain assembled, then is possible to obtain a close system. 
In the overlapping approach, the problem can be solved by an iterative scheme in terms of one of the Schwarz methods, i.e. by solving recursively each of the sub-domains, or instead the complete close system can be solved directly, after imposing the interface matching conditions between subdomains, without the used of an iterative scheme.

It is important to observe that the non-overlapping domain decomposition approach is naturally suited for the numerical solution of multi-zone problems, where the governing equations have different values of the problem parameters at different regions of the problem domain.

Several application of the domain decomposition approach has been reported in the literature when solving partial differential equations with the use of the Unsymmetric radial basis function collocation approach. In the work by Kansa and Carlson [13] they conclude that one of the most efficient technique when solving dense system of linear equation is to use preconditioning and to make use of domain decomposition techniques.

In this work will be implement the non-overlapping non-iterative domain decomposition approach for the numerical solution of boundary values problems based on the symmetric radial basis function collocation approach, with application to multi-zone problems.

\section{Multi-zone problems}

Considering a problem that contains different regions, in which the coefficients of the governing equation are constant but different in each of them. In the implementation of the non-overlapping domain decomposition approach for the solution of multi-zone problems, the problem's domain is divided into a finite number of non-overlapping zones according with the behaviour of the governing equation.

In order to implement the symmetric approach to solve this type of multi-zone domain problem, the solution at each zone is represented by its corresponding symmetric interpolation using a set of collocation points within each of the zones and the points at the interface between them. At the interfaces points that coincide with the physical boundary of the problem, the corresponding boundary conditions are imposed, while at the internal points, it is required that the governing equation, with corresponding value of the parameters at each zone, should be satisfy.

To solve this type of problem it is necessary to impose the continuity of flux at the interfaces between the zones, i.e. the flux leaving one sub-zone has to be equal to the flux entering the other. Therefore, it is necessary that the following flux matching conditions hold at the $m^{\text {th }}$ interface of the sub-zones $i$ and $i+1$ :

$$
\left[D^{i} \frac{\partial C^{i}}{\partial n}-C^{i} u_{j}^{i} n_{j}\right]_{m}=\left[D^{i+1} \frac{\partial C^{i+1}}{\partial n}-C^{i+1} u_{j}^{i+1} n_{j}\right]_{m}
$$

Besides the above conditions, the concentration at each interface needs to be continuous, i.e.: 


$$
\left[C^{i}\right]_{m}=\left[C^{i+1}\right]_{m}
$$

The Hermitian interpolation property of the symmetric approach (which takes into account the function and it's derivate) makes this method a natural technique to deal with the above matching conditions. There are two different alternatives to impose the interface matching conditions. First two different set of points at each sub-domains interface are defined. In each set of points a different matching condition is imposed, i.e. of the $n_{i}=n_{i 1}+n_{i 2}$ interface points $n_{i 1}$ are required to satisfy the continuity of the concentration and on the remaining $n_{i 2}$ points the flux matching condition is satisfied. On the other hand, due to the dependence of the Hermite interpolation on the partial differential operators, it is possible to impose simultaneously both conditions at each interface point. In this last case, as we are using a Hermite interpolation scheme, the resulting matrix system is non-singular as long as the partial differential operators applied to each point are linearly independent, even if in a single node we impose two different differential conditions (see Wu [11]). In this case, at each interface point both matching conditions, i.e. concentration and flux, are required to be satisfied. Therefore, the Hermite interpolation function is represented by:

$$
\begin{aligned}
C(x)= & \sum_{k=1}^{n_{b}} \lambda_{k} B_{\xi} \Psi\left(\left\|x-\xi_{k}\right\|\right)+\sum_{k=n_{b}+1}^{n_{i}} \lambda_{k} \Psi\left(\left\|x-\xi_{k}\right\|\right)+\sum_{k=n_{b}+n_{i}+1}^{n_{b}+2 n_{i}} \lambda_{k} \\
& \left(D^{i} \frac{\partial}{\partial n_{\xi}} \Psi\left(\left\|x-\xi_{k}\right\|\right)\right)+\sum_{k=n_{b}+2 n_{i}}^{N} \lambda_{k} L_{\xi}^{i} \Psi\left(\left\|x-\xi_{k}\right\|\right)+P_{m-1}(x)
\end{aligned}
$$

with $n_{b}$ as the number of nodes on the boundary of a subdomain that coincide with the physical boundary, i.e. at an internal subdomain $n_{b} \equiv 0, n_{i}$ the number of nodes on the interfaces common with other subdomains and $N-\left(n_{b}+n_{i}\right)$ the number of internal nodes at the subdomain. As before, in the above expression $L_{\xi}^{i}$ and $B \xi$ are the differential operators corresponding to the partial differential equation at the subdomain and the boundary differential operator.

In the above interpolation function, the flux matching condition (9) at the interface $m$ is reduced to

$$
\left[D^{i} \frac{\partial C^{i}}{\partial n}\right]_{m}=\left[D^{i+1} \frac{\partial C^{i+1}}{\partial n}\right]_{m}
$$

since we are imposing simultaneously at each interface point the continuity of concentration and flux, besides the convective velocity field needs to be continuous across the sub-domains.

It is important to point out that the above double collocation strategy at the interface points can also be used in the standard Unsymmetric approach (Kansa method). However due to lack of dependence on the differential operators of the 
corresponding interpolation function, this alternative will results in an overdetermined system of algebraic equations.

\subsection{Numerical examples}

Let us consider the steady state heat transfer problem in a circular cylinder with a circular hollow. At the origin a constant value of temperature is given as well as at the exterior wall. The cylinder consists of three rings of constant but different parameters (see figure 1), under these conditions, in cylindrical co-ordinates, the problem is described by the following equation:

$$
\frac{K^{i}}{r} \frac{d}{d r}\left(r \frac{d T^{i}}{d r}\right)+\alpha^{i}=0 \quad i=1,2,3
$$

where $K^{i}$ and $\alpha^{i}$ are the thermal conductivity and heat generation rate at the zone (ring) $\mathrm{i}$, respectively, and $\mathrm{r}$ is the radial distance. The matching conditions at the contact region between rings, i.e. continuity of temperature and flux are given by:

$$
T^{i}=T^{i+1} \text { and } \frac{K^{i}}{r} \frac{d}{d r}\left(r T^{i}\right)=\frac{K^{i+1}}{r} \frac{d}{d r}\left(r T^{i+1}\right) \text { at } r=r_{m}, m=1,2,3
$$

The analytical solution of the problem is given by Carslaw and Jaeger [13].

By expanding the cylindrical Laplacian operator in equation (21), we obtain the following expression:

$$
K^{i} \frac{d^{2} T^{i}}{d r^{2}}+\frac{K^{i}}{r} \frac{d T^{i}}{d r}+\alpha^{i}=0
$$

which can be interpreted as a one-dimensional convection diffusion equation with variable negative convective velocity field, $u_{r}^{i}=-K^{i} / r$.

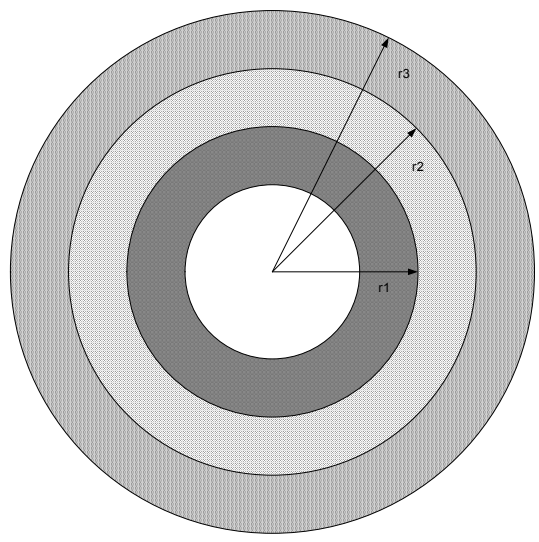

Figure 1: Cylindrical domain consisting of three rings with different constant coefficients at each ring. 
The one-dimensional problem defined by equation (15), the matching conditions given by equation (14) and the corresponding boundary conditions at $r=r_{0}$ and $r=r_{3}$, will be solve here as two dimensional convection diffusion problem in the rectangular domain $1 \leq x \leq r_{3}=7 d m$ and $0 \leq y \leq 2 d m$ with zero lateral flux, were at each zone (ring) the following governing equation is satisfied:

$$
K^{i} \frac{\partial^{2} T^{i}}{\partial x_{j}^{2}}-u_{1}^{i} \frac{\partial T^{i}}{\partial x_{1}}+\alpha^{i}=0 \text { where } u_{1}^{i}=-K^{i} / x_{1}
$$

Two cases are considered, with different parameters in each zone and the same boundary conditions; $T(0, y)=1, T(7, y)=2$ and $\partial T / \partial n=0$ at $y=0$ and $y=2$.

In figures 2 and 3, it is possible to appreciate the excellent agreement found between the numerical results using the above symmetric meshless collocation method and the analytical solution. In the first example (figure 2), a total of 841 collocation points uniformly distributed were used in order to achieve the obtained accuracy. The second case is more computational demanding due to the drastic changes in the heat production term, $\alpha^{i}(i=1,2,3)$, between the different zones, given by $\alpha^{1}=5, \alpha^{2}=-3, \alpha^{3}=10$ (temperature/sec). In this case, we compare how the numerical result is affect by increasing the total number of collocation points. In figure 3, the results for two different set of uniform distribution of collocation points (841 and 2987) are presented, showing the convergence of the numerical scheme.

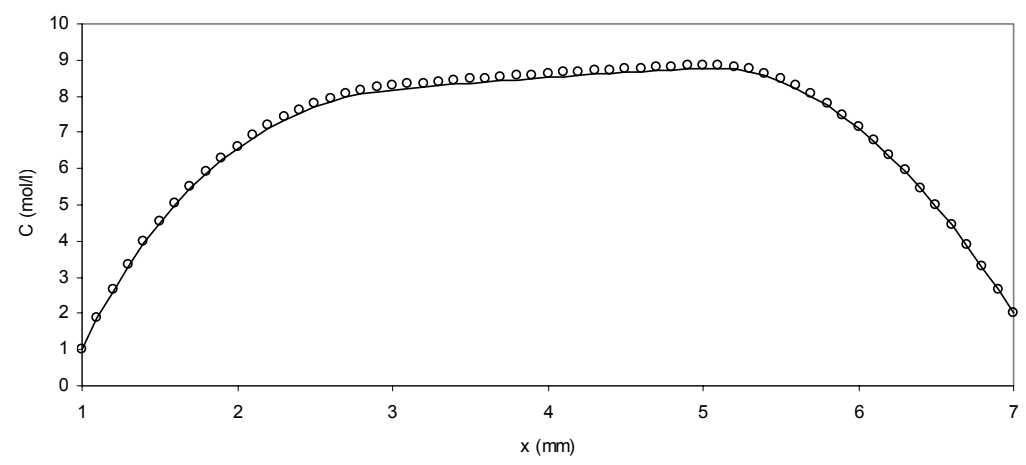

Figure 2: Comparison between the analytical solution - and the numerical results $\circ$ for the following parameter: $\alpha^{1}=2, \alpha^{2}=0, \alpha^{3}=4$ (temperature/sec) and $K^{1}=K^{2}=K^{3}=1\left(d^{2} / \mathrm{sec}\right)$. 


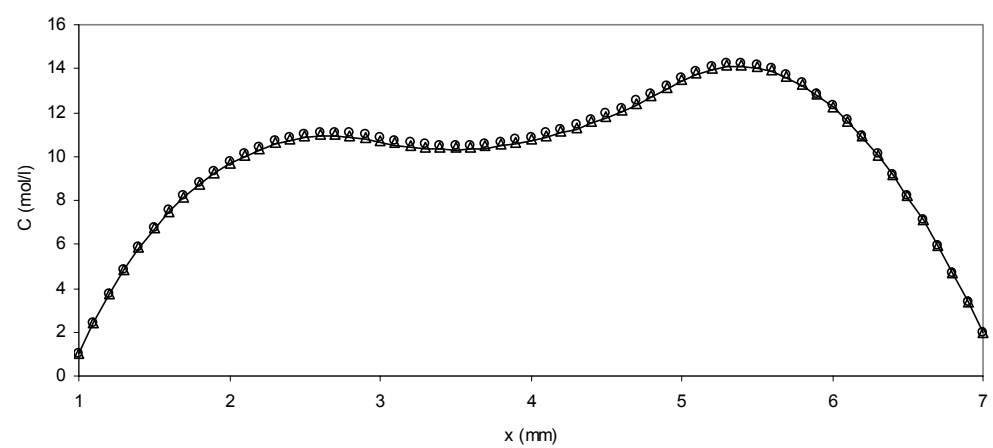

Figure 3: Comparison between the analytical solution - and the numerical results obtained with a total of $841,\left(^{\circ}\right)$, and $2987,(\Delta)$, collocation points, for the following parameters: $\alpha^{1}=5, \alpha^{2}=-3, \alpha^{3}=10 \quad$ (temperature/sec) and $K^{1}=K^{2}=K^{3}=1\left(d m^{2} / \mathrm{sec}\right)$.

\section{Conclusions}

The use of symmetric radial basis function collocation method to solve partial differential equations provides a simply accurate and truly meshes free technique. It is important to point out that in the case when this scheme is applied to solve large scale problems with a large number of data points, the conditional number of the resulting collocation matrix could be very large and the computational performance poor. As a way to overcome these problems, a domain decomposition scheme with double collocation at the interfaces joining neighbouring sub-domains is proposed. The proposed domain decomposition technique makes possible to improve the ill-conditioning problem through the reduction of the size of the full coefficient matrix to be solved in a global manner.

\section{Acknowledgements}

This research was been partially sponsored by the GABARDINE project (Contract number 518118) - part of the FP6-2006-TTC-TU European Commission Programme.

\section{References}

[1] E.J.Kansa, Multiquadrics- A scattered data approximation scheme with applications to computation fluid-dynamics-I: Surface approximations and partial derivatives estimates; Computers Math. Applic. 19, pp 127-145, (1990) 
[2] E.J.Kansa, Multiquadrics- A scattered data approximation scheme with applications to computation fluid-dynamics-II: Solution to parabolic, hyperbolic and elliptic partial differential equations; Computers Math. Applic. 19, pp 147-161, (1990)

[3] Dubal M.R. Domain decomposition and local refinement for multiquadric approximations. I: second-order equations in one-dimension, Journal of Applied Science, 1, No.1, 146-171 (1994).

[4] Y. C. Hon and X. Z. Mao An efficient numerical scheme for Burgers' equations, Appl. Math. and Comp. 95, 37-50 (1998).

[5] Z Zerroukat M., Power H. and Chen C.S., A numerical method for heat transfer problems using collocation and radial basis functions, Int. J. Numer. Meth. Engng, 42, 1263-1279, (1998).

[6] Dubal M.R., Olivera S.R. and Matzner R.A. In Approaches to Numerical Relativity, Editors: R.d Inverno, Cambridge University Press, Cambridge UK, (1993).

[7] Kansa E.J. and Hon Y.C., Circumventing the ill conditioning problem with multiquadric radial basis functions: applications to elliptic partial differential equations, 39, 123-137, (2000).

[8] Fedoseyev AI, Friedmann MJ, Kansa EJ. Improved multiquadratic method for elliptic partial differential equation via PDE collocation on the boundary. Comput. Math. Appl. 2002, 43, 439-455

[9] Fasshauer G.E. Solving Partial Differential Equations by Collocation with Radial Basis functions, Proceedings of Chamonix, Editors: A. Le Méchauté, C. Rabut and L.L. Schumaker, 1-8, Vanderbilt University Press, Nashville, TN (1996).

[10] Wu Z., Hermite-Birkhoff interpolation of scattered data by radial basis functions; Approx. Theory, 8:2, 1-11 (1992).

[11] Wu Z., Solving PDE with radial basis function and the error estimation; Advances in Computational Mathematics, Lecture Notes on Pure and Applied Mathematics, 202, Editors: Z. Chen, Y. Li, C.A. Micchelli, Y. Xu and M. Dekker, GuangZhou (1998).

[12] Schaback R and Franke C., 'Covergence order estimates of meshless collocation methods using radial basis functions', Advances Computational Mathematics, 8, Issue 4, 381-399, (1998).

[13] Kansa E.J. \& Carlson 1992, 'Improved accuracy of multiquadric interpolation using variable shape parameters', Computers \& Mathematic with Application, vol. 24, 99-120.

[14] Carslaw H.S. and Jaeger J.C., Conduction of heat in solids, Oxford at the Clarendon press, Oxford, (1959). 